\title{
Fucose-containing exopolysaccharide produced by the newly isolated Enterobacter strain A47 DSM 23139
}

\author{
Filomena Freitas $^{\mathrm{a}, *}$, Vitor D. Alves ${ }^{\mathrm{b}}$, Cristiana A.V. Torres ${ }^{\mathrm{a}}$, Madalena Cruz $^{\mathrm{a}}$, \\ Isabel Sousa $^{b}$, Maria João Melo ${ }^{a}$, Ana M. Ramos ${ }^{a}$, Maria A.M. Reis ${ }^{a}$ \\ a REQUIMTE/CQFB, Chemistry Department, FCT/Universidade Nova de Lisboa, 2829-516 Caparica, Portugal \\ b CEER-Biosystems Engineering, ISA/Universidade Técnica de Lisboa, Tapada da Ajuda, 1349-017 Lisboa, Portugal
}

\section{A R T I C L E I N F O}

Article history:

Received 30 April 2010

Received in revised form 15 June 2010

Accepted 16 July 2010

Available online 23 July 2010

\section{Keywords:}

Fucose

Exopolysaccharide

Rheology

Emulsifying activity

Flocculating activity

\begin{abstract}
A B S T R A C T
Enterobacter strain A47 (DSM 23139) was found to produce a fucose-containing exopolysaccharide (EPS) The EPS is composed of fucose, galactose, glucose, pyruvate, succinate and acetate in the molar ratios 1.6:1.3:1.1:1.2:0.7:1.5. It is a high molecular weight $\left(5.8 \times 10^{6}\right)$ homogeneous biopolymer, as indicated by the low polydispersity value (1.3). The steady shear flow properties of the EPS aqueous solutions are similar to guar gum and fucogel, and its viscoelastic properties indicate the formation of viscous aqueous solutions with entangled polymer chains. Additionally, the EPS has demonstrated good flocculating and emulsion stabilizing capacities, comparable to some commercially available products. These functional properties make the fucose-containing EPS a good alternative to many synthetic polymers, as well as other natural polysaccharides, in several applications in the food, pharmaceutical, cosmetic, textile, paper and petroleum industries.
\end{abstract}

(c) 2010 Elsevier Ltd. All rights reserved.

\section{Introduction}

Fucose-containing polysaccharides, in addition to their thickening, emulsifying and/or film-forming properties, have increased market value because fucose is one of the rare sugars, difficult to obtain, with many applications that include cosmetics and pharmaceuticals (Vanhooren \& Vandamme, 1999). Preparations containing fucose, fucose-containing oligo- and polysaccharides were reported to have biological properties that potentiate their therapeutic use, for example, as anti-carcinogenic and antiinflammatory agents, in the treatment of rheumatoid arthritis, in age-related pathologies accompanied by tissue loss, in acceleration of wound healing and as hydrating and anti-aging additives (Cescutti et al., 2005; Péterszegi, Fodil-Bourahla, Robert, \& Robert, 2003).

Oligo- and polysaccharides containing fucose may be found in microorganisms, plants, seaweeds and animals (Vanhooren \& Vandamme, 1999). Microorganisms producing exopolysaccharides rich in fucose include a wide range of bacteria, fungi and microalgae. Microbial production of such biopolymers guarantees an easy and fast mass production, unaffected by environmental factors. More-

\footnotetext{
* Corresponding author at: Departamento de Química, Laboratório de Engenharia Bioquímica, Faculdade de Ciências e Tecnologia, Universidade Nova de Lisboa, 2825516 Caparica, Portugal, Tel.: +351 21 2948357; fax: +351212948385.

E-mail address: filomenafreitas@dq.fct.unl.pt (F. Freitas).
}

over, microorganisms are generally better suited for polysaccharide production than plant or algae, exhibiting higher growth rates and being more amenable to manipulation of conditions for enhancing growth and/or production (Moreno, Vargas, Olivares, Rivas, \& Guerrero, 1998).

Extensively studied bacterial fucose-containing extracellular polysaccharides include colanic acid, fucogel and clavan. Colanic acid is a polysaccharide composed of fucose, glucose, galactose and glucuronic acid, and the acyl groups pyruvyl and acetyl (Grant, Sutherland, \& Wilkinson, 1969). It is commonly produced by many members of the family Enterobacteriaceae, including Escherichia, Salmonella and Klebsiella strains (Ratto et al., 2006). Fucogel is a polysaccharide produced by Klebsiella pneumoniae I-1507, composed of galactose, 4-O-acetyl-galacturonic acid and fucose (Guetta, Mazeau, Auzely, Milas, \& Rinaudo, 2003). It has been successfully commercialized by Solabia BioEurope, France, for the cosmetic industry (Paul et al., 1996). Clavan is composed of glucose, galactose, fucose and pyruvyl, being produced by Clavibacter michiganensis strains (van den Bulk, Zevenhuizen, Cordewener, \& Dons, 1991; Vanhooren \& Vandamme, 2000).

Among the genus Enterobacter, several species have been reported to secrete exopolysaccharides containing fucose. Examples include: Enterobacter sp. CNCM 1-2744 that produces an EPS in which fucose, galactose, glucose and glucuronic acid monomers are present in a ratio of 2:2:1:1 (Philbe, 2002), Enterobacter sp. SSYL (KCTC 0687BP) that produces an EPS in which fucose represents $8-10 \%$ of the sugar content, being glucuronic acid the main compo- 
nent (40-70\%) (Yang, 2002), E. sakazakii, strains ATCC 53017, ATCC 29004 and ATCC 12868 that produce an EPS in which fucose represents $13-22 \%$ (Harris \& Oriel, 1989) and E. amnigenus that produces a heteropolymer containing glucose, galactose, fucose, mannose, glucuronic acid and pyruvil (Cescutti et al., 2005).

Previously, we reported that in a nutrient medium containing glycerol byproduct from the biodiesel industry as the sole carbon source, an Enterobacter strain produced an exopolysaccharide (EPS) composed of glucose, galactose and fucose (Alves et al., 2010). In the present work, a preliminary polymer characterization in terms of its chemical composition, molecular weight and intrinsic viscosity is presented. The functional properties of this fucose-containing EPS, including its rheological behavior in aqueous medium, emulsion forming and stabilizing capacity and flocculating activity, were compared to other commercially available polymers, namely, fucogel, xanthan gum, guar gum, alginate, pectin and carboxymethylcellulose (CMC), to evaluate its potential industrial applications.

\section{Material and methods}

\subsection{Production of the fucose-containing EPS}

The strain used in this work was isolated from a contaminated glycerol byproduct aqueous solution. A morphological, physiological and genetic characterization of the strain was performed by the Deutsche Sammlung von Mikroorganismen und Zellkulturen (DSMZ), Germany, according to the Standard Methods. The strain was named Enterobacter strain A47 and deposited at DSMZ under accession number 23139.

Enterobacter strain A47 was cultivated using glycerol byproduct (supplied by SGC Energia, SGPS, SA, Portugal) as the sole carbon source in a $10 \mathrm{~L}$ bioreactor (BioStat B-plus, Sartorius), with controlled temperature and $\mathrm{pH}$ of $30.0 \pm 0.1^{\circ} \mathrm{C}$ and $6.80 \pm 0.05$, respectively. The bioreactor was operated in a batch mode during the first day of cultivation and, afterwards, in a fed-batch mode, by supplying the bioreactor with cultivation Medium $E^{*}$, with a glycerol concentration of $200 \mathrm{~g} \mathrm{~L}^{-1}$, at a constant rate of $20 \mathrm{~mL} \mathrm{~h}^{-1}$. The aeration rate $(0.125 \mathrm{vvm}$, volume of air per volume of reactor per minute) was kept constant throughout the cultivation, and the dissolved oxygen concentration (DO) was controlled by automatic variation of the stirrer speed ( $400-800 \mathrm{rpm}$ ) provided by two 6-blade impellers. During the fed-batch phase, the DO was maintained below $10 \%$.

The culture broth recovered from the bioreactor at the end of the cultivation was diluted $(1: 2, \mathrm{v} / \mathrm{v})$ for viscosity reduction and the bacterial cells were removed by centrifugation $(13,000 \times g$, $1 \mathrm{~h})$. The cell-free supernatant was subjected to thermal treatment $\left(70^{\circ} \mathrm{C}, 1 \mathrm{~h}\right)$ to inactivate bacterial enzymes that might cause polymer degradation during the subsequent purification steps. The treated supernatant was centrifuged $(13,000 \times g, 1 \mathrm{~h})$ to remove any remaining cell debris and denatured proteins. Finally, it was dialyzed with a 10,000 MWCO membrane (SnakeSkin ${ }^{\mathrm{TM}}$ Pleated Dialysis Tubing, Thermo Scientific), against deionized water ( $48 \mathrm{~h}$, $4{ }^{\circ} \mathrm{C}$ ) and freeze dried.

\subsection{Characterization of the fucose-containing EPS}

\subsubsection{Chemical composition}

The EPS was analyzed in terms of its sugar composition, acyl groups, inorganic and protein content. For the analysis of the sugar composition, polysaccharide samples $(\sim 5 \mathrm{mg})$ were dissolved in dionized water $(5 \mathrm{~mL})$ and hydrolyzed with trifluoroacetic acid (TFA) $(0.1 \mathrm{~mL}$ TFA $99 \%)$, at $120^{\circ} \mathrm{C}$, for $2 \mathrm{~h}$. The hydrolysate was used for the identification and quantification of the constituent monosaccharides and the acyl groups substituents present in the biopolymer by HPLC, as described by Freitas, Alves, Pais et al. (2009). Total protein content was determined by the modified Lowry method, as described by Hilliou et al. (2009). The total inorganic content of the biopolymer was evaluated by subjecting it to pyrolysis at a temperature of $550^{\circ} \mathrm{C}$ for $48 \mathrm{~h}$.

\subsubsection{FT-IR spectroscopy}

The infrared spectra of the polymers were acquired with a Nicolet Nexus spectrophotometer interfaced with a Continumm microscope, using a MCT-A detector cooled by liquid nitrogen. All the spectra presented were obtained in transmission mode, using a Thermo diamond anvil compression cell. The spectra were obtained, in a $100 \mu \mathrm{m} \times 100 \mu \mathrm{m}$ area, with a resolution of $4 \mathrm{~cm}^{-1}$ $\left(8 \mathrm{~cm}^{-1}\right.$ in the exopolysaccharide analysis) and 128 scans. They are shown here as acquired, without corrections or any further manipulations, except for the removal of the $\mathrm{CO}_{2}$ absorption at approximately $2300-2400 \mathrm{~cm}^{-1}$.

\subsubsection{Average molecular weight}

Number and weight average molecular weights $\left(M_{\mathrm{n}}\right.$ and $M_{\mathrm{w}}$, respectively), as well as the polydispersity index $\left(\mathrm{PD}=M_{\mathrm{w}} / M_{\mathrm{n}}\right)$ were obtained by size exclusion chromatography (SEC) in a low temperature Waters Co. apparatus, equipped with a Waters Ultrahydrogel Linear column and a differential refractive index detector (Waters 2410). A $0.1 \mathrm{M} \mathrm{NaCl}$ solution at $30^{\circ} \mathrm{C}$ was used as eluent, and the polymer concentration was less than $0.1 \mathrm{wt} . \%$, thus ensuring the pumping of essentially non aggregated polysaccharides in coil conformation by a Waters 510 Solvent Delivery System. The values of $M_{\mathrm{w}}$ and $M_{\mathrm{n}}$ were calculated using a relative calibration curve generated with monodisperse pullulan standards (Shodex, Showa Denko, Japan).

\subsubsection{Intrinsic viscosity}

The intrinsic viscosity of the purified polymer was determined by double extrapolation to zero concentration of the Huggins and Kraemer equations, respectively (Rao, 1999):

$$
\begin{aligned}
& \frac{\eta_{\mathrm{sp}}}{C}=[\eta]+k_{\mathrm{H}}[\eta]^{2} \mathrm{C} \\
& \frac{\ln \left(\eta_{\mathrm{rel}}\right)}{C}=[\eta]+k_{\mathrm{k}}[\eta]^{2} C
\end{aligned}
$$

where $[\eta], \eta_{\mathrm{sp}}$ and $\eta_{\text {rel }}$ are the intrinsic, specific and relative viscosities, respectively; $k_{\mathrm{H}}$ and $k_{\mathrm{k}}$ are the Huggins and Kraemer coefficients, and $C$ is the polymer concentration. An automatic viscosity measuring unit AVS 450 (Schott-Gerate, Germany), with an Ubbelhode capillary viscometer (Ref. 53013/Ic, Schott-Gerate, Germany) was used to measure the viscosity of dilute solutions at $25^{\circ} \mathrm{C}$, with a relative viscosity in the range between 1.2 and 2.0 in order to ensure a good accuracy in the extrapolations to zero concentration.

\subsection{Rheological properties in aqueous medium}

The rheology of polymers aqueous solutions were studied using a controlled stress rheometer (Haake RS-75, Germany), equipped with a cone and plate geometry (diameter $3.5 \mathrm{~cm}$, angle $2^{\circ}$ ). During the experiments, the shearing geometry was covered with paraffin oil in order to prevent water loss. Flow curves were determined using a steady state flow ramp in the shear range of $1-700 \mathrm{~s}^{-1}$. The shear rate was measured point by point with consecutive $30 \mathrm{~s}$ steps of constant shear rate. The viscosity was recorded for each point to obtain the flow curves. Frequency sweeps were carried at out at a controlled stress of $1 \mathrm{~Pa}$ (shown by stress sweeps to give values within the linear viscoelastic region) in order to measure the dynamic moduli $G^{\prime}$ and $G^{\prime \prime}$. 


\subsection{Emulsifying activity}

The capacity of the EPS to stabilize emulsions with several hydrophobic compounds was tested as described by Freitas, Alves, Carvalheira et al. (2009). Briefly, an EPS aqueous solution (0.5 wt.\%) was mixed with each hydrophobic compound (3:2, v/v ratio) and stirred in the vortex, and the emulsification index after $24 \mathrm{~h}\left(E_{24}\right)$ was determined, using the following equation:

$E_{24}=\frac{h_{\mathrm{e}}}{h_{\mathrm{T}}} \times 100$

where $h_{\mathrm{e}}(\mathrm{mm})$ is the height of the emulsion layer and $h_{\mathrm{T}}(\mathrm{mm})$ is the overall height of the mixture. All tests were performed in duplicate. The tested compounds included hydrocarbons (hexane, Riedel de Haen; hexadecane, Sigma; toluene, Sigma; diethylether, Riedel de Haen; benzene, Riedel de Haen) and oils (corn oil, sunflower oil, rice bran oil and cedar wood oil, purchased at the local supermarket; and silicone oil, Wacker).

The test was also carried out for fucogel (Solabia), xanthan gum (Fluka), citrus pectin (Riedel de Haen), guar gum (Fluka), sodium alginate (Sigma), carboxymethylcelullose (Aqualon, France) and Triton X-100 (Riedel de Haen), with the same concentration in deionized water.

\subsection{Flocculating activity}

The flocculating activity was tested using kaolin clay (Fluka) as the suspended solid (Gong et al., 2008; Kurane, Takeda, \& Suzuki, 1986) for an EPS concentration of $0.01 \mathrm{wt} . \%$. The test was also carried out using aqueous solutions of several commercial polysaccharides, namely, fucogel (Solabia), xanthan gum (Fluka), citrus pectin (Riedel de Haen), guar gum (Fluka), sodium alginate (Sigma) and carboxymethylcelullose (Aqualon, France) with the same concentration. An inorganic flocculant, $\mathrm{Al}_{2}\left(\mathrm{SO}_{4}\right)_{3}$ (Riedel de Haen), was also tested at the same concentration. All tests were performed in duplicate.

\section{Results and discussion}

\subsection{Production of the fucose-containing EPS}

Enterobacter strain A47 was cultivated in a nutrient medium containing glycerol from the biodiesel industry as the sole carbon source for the production of the fucose-containing EPS. In a 7 days cultivation run, $24.34 \mathrm{~g} / \mathrm{L}$ of glycerol were consumed and $13.3 \mathrm{~g} / \mathrm{L}$ of polymer were obtained by acetone precipitation (Alves et al., 2010). In the present work, with the objective of obtaining a refined polymer, the EPS was extracted by dialysis of the cell-free supernatant. This procedure allowed for the elimination of remnants of the culture broth, namely, proteins and salts, which co-precipitated with the polysaccharide during the acetone extraction procedure (Alves et al., 2010). Hence, $8.1 \mathrm{~g} / \mathrm{L}$ of pure EPS were obtained through dialysis of the cell-free supernatant. Moreover, in addition to yielding a more refined biopolymer, this procedure also had the advantage of being a solvent-free extraction method.

Concomitant with EPS synthesis, there was a considerable viscosity built up and the cultivation broth developed a nonNewtonian behavior, showing an increase of shear-thinning as the cultivation time proceeded (Alves et al., 2010). The apparent viscosity of the culture broth measured at low shear rates has shown an increase of two orders of magnitude (from $10^{-3}$ to $10^{-1}$ Pa s) (Alves et al., 2010). This viscosity built up is a common feature observed in many microbial cultivations for the production of extracellular polysaccharides and it usually determines the termination of the run due to loss of bulk homogeneity of the culture broth (Freitas et al., 2009b).

\subsection{Characterization of the fucose-containing EPS}

\subsubsection{Chemical composition}

As described previously, the EPS was composed of fucose, galactose and glucose, and acyl substituents (pyruvate, acetate and succinate) (Alves et al., 2010). The acetone extracted polymer contained considerable amounts of contaminants, namely, proteins (7.6-15.9 wt.\%) and inorganic residues (32.5\%), remnants of the culture broth that co-precipitated with the polysaccharide (Alves et al., 2010). In this study, a refined polymer was obtained by dialysis, with a much reduced amount of contaminants. In fact, no inorganic residue was observed after the polymer being subjected to pyrolysis for $24 \mathrm{~h}$. A fraction of the protein content still remained in the dialyzed polymer ( $9.1 \mathrm{wt} . \%)$, suggesting that it might be composed of high molecular weight molecules. The protein content of the polymer was further reduced to $<5 \mathrm{wt}$.\% by using a $50,000 \mathrm{MWCO}$ dialysis membrane.

The resulting purified EPS was composed of fucose, galactose, glucose, pyruvate, succinate and acetate in the molar ratios 1.6:1.3:1.1:1.2:0.7:1.5. Fucose, galactose and glucose are neutral sugar residues commonly found in exopolysaccharides produced by members of the family Enterobacteriaceae (Ratto et al., 2006). Nevertheless, in contrast with the fucose-containing EPS of the present study, all those polymers have uronic acids (glucuronic or galacturonic acids) as main components. Acetate esters, pyruvate ketals and succinyl half esters are common non-sugar components of microbial polysaccharides, which greatly influence polymer properties, namely, solubility and rheology (Rinaudo, 2004). Moreover, the presence of pyruvate and succinate give the EPS an anionic character (Freitas et al., 2009a).

\subsubsection{Fourier transform infrared spectroscopy}

The FT-IR spectrum of the fucose-containing EPS is presented in Fig. 1 along with the spectra of several other commercial polysaccharides for comparison. The polysaccharides analyzed were: fucogel, which is a bacterial fucose-containing EPS, composed by fucose, galactose and galacturonic acid; alginate, which is an algae polysaccharide, composed by mannuronic and guluronic acids, and acetate); and guar gum (a neutral plant polysaccharide composed of galactose and mannose).

The broad and intense band around $3400 \mathrm{~cm}^{-1}$, common to all polysaccharides, represents $\mathrm{O}-\mathrm{H}$ stretching of hydroxyls and bound water (Synytsya, Copikova, Matejka, \& Machovic, 2003), which overlaps in part with the $\mathrm{C}-\mathrm{H}$ stretching peak of $\mathrm{CH}_{2}$ groups appearing at $2940 \mathrm{~cm}^{-1}$. The well-defined envelope found between 1200 and $900 \mathrm{~cm}^{-1}$ represents skeletal C-O and C-C vibration bands of glycosidic bonds and pyranoid ring (Synytsya et al., 2003).

The band at $1720 \mathrm{~cm}^{-1}$ observed in the fucose-containing EPS and fucogel spectra, but not identified in guar gum nor Alginate, may be attributed to the $\mathrm{C}=\mathrm{O}$ stretching of carbonyls in acyl groups (Alvarez-Mancenido, Landin, Lacik, \& Martínez-Pacheco, 2008). Similarly, the band at $1250 \mathrm{~cm}^{-1}$ may also be attributed to the C-O-C vibration of acyls (Synytsya et al., 2003). The two strong bands around 1607 and $1405 \mathrm{~cm}^{-1}$ in alginate spectrum (Fig. 1(c)), which can be attributed to the asymmetric and symmetric stretchings of carboxylates, respectively (Synytsya et al., 2003), are also observed in fucogel and the fucose-containing EPS spectra (Fig. 1(a) and (b)). Those bands are not seen in guar gum (Fig. 1(d)), since this polysaccharide is a neutral polymer. Even though the fucose-containing EPS and fucogel spectra are quite similar, there is a distinctive band at $1564 \mathrm{~cm}^{-1}$ observed for the fucose-containing EPS (Fig. 1(a)) that may be attributed to the $\mathrm{C}=\mathrm{O}$ anti-symmetric stretching vibrations of succinate (Krishnan, Raj, Robert, Ramanand, \& Das, 2007). The identification of bands corresponding to acid groups in the fucose-containing EPS spectrum 


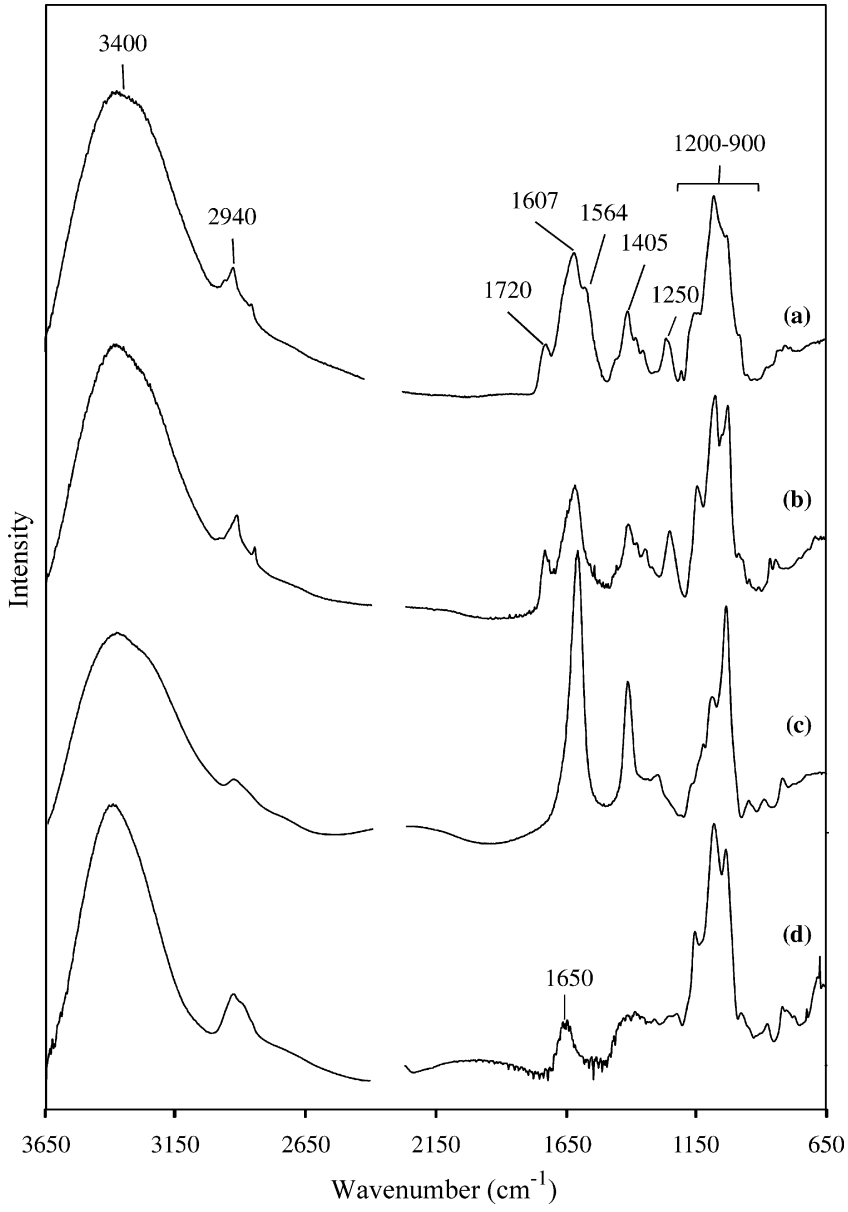

Fig. 1. Comparative FT-IR spectra of polysaccharides: fucose-containing EPS (a), fucogel (b), alginate (c) and guar gum (d).

is consistent with the presence of acyl groups, which account for $22.4 \mathrm{wt}$.\% of the polymer.

\subsubsection{Molecular weight}

The average molecular weight $\left(M_{\mathrm{w}}\right)$ and the polydispersity (PD) of the fucose-containing EPS produced by Enterobacter strain A47 were determined by size exclusion chromatography (SEC). For comparison, several commercial polysaccharides from different sources were also analyzed by SEC, namely, bacterial (xanthan and fucogel), plant (guar gum and citrus pectin), algae (alginate) and semi-synthetic (carboxymethylcellulose). The $M_{\mathrm{w}}$ of the EPS was calculated to be $5.8 \times 10^{6}$. This value is comparable to the $M_{\mathrm{w}}$ of xanthan $\left(5.0 \times 10^{6}\right)$, fucogel $\left(3.1 \times 10^{6}\right)$ and guar gum $\left(2.6 \times 10^{6}\right)$. The $M_{\mathrm{w}}$ of the other polysaccharides analyzed was one order of magnitude lower: $5.1 \times 10^{5}$, for CMC; $2.2 \times 10^{5}$, for pectin; and $4.3 \times 10^{5}$, for alginate. Although the presented $M_{\mathrm{w}}$ values are not absolute their order of magnitude and their comparison, as well, shall be valid as they were calculated based on a calibration curve generated with polysaccharide standards.

The $M_{\mathrm{W}}$ and the PD are important parameters that will determine the suitability of a given polymer for specific applications. The PD reflects the degree of heterogeneity of the polymer's chain lengths. Comparing to the commercial polysaccharides analyzed, the EPS was the most homogeneous, with a PD of 1.3, while xanthan, fucogel and guar gum had PD values between 2.4 and 2.9. PD values are absolute, as they represent a ratio between average molecular weights.

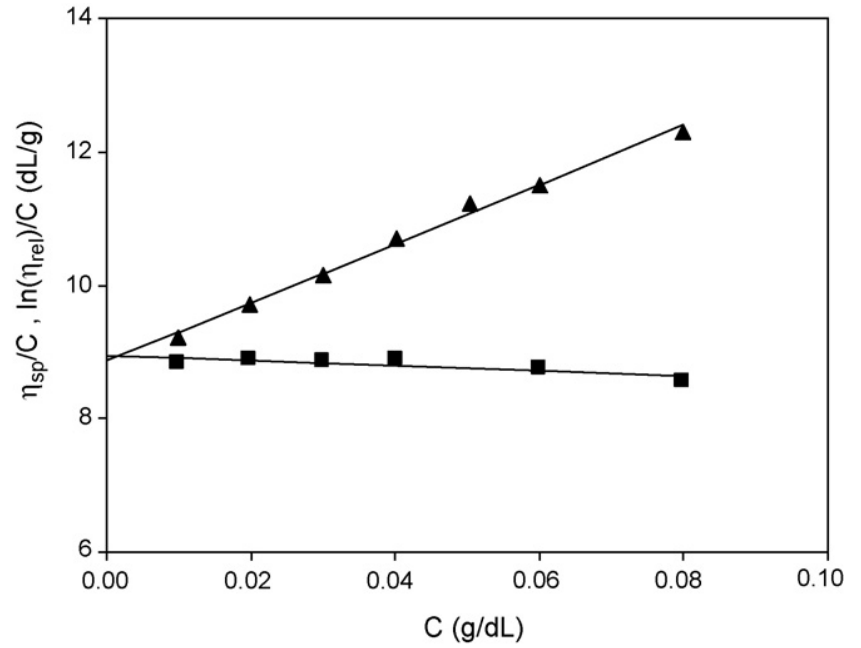

Fig. 2. Evaluation of the intrinsic viscosity in $0.1 \mathrm{M} \mathrm{NaCl}$ using Huggins ( $\mathbf{\Delta}$ ) and Kraemer ( $\mathbf{\square})$ equations.

\subsubsection{Intrinsic viscosity}

The intrinsic viscosity is a characteristic property of a single macromolecule in a given solvent and is a measure of the hydrodynamic volume occupied by the polymer itself. It depends on the polymer's molecular mass, chain rigidity and type of solvent. The value obtained for the fucose-containing EPS produced by Enterobacter strain A47, in $0.1 \mathrm{M} \mathrm{NaCl}$ at $25^{\circ} \mathrm{C}$, by double extrapolation to zero concentration of the Huggins and Kraemer equations was $8.9 \mathrm{dL} / \mathrm{g}$ (Fig. 2). This value is lower than that presented in a previous study $(11.4 \mathrm{dL} / \mathrm{g}$ ) (Alves et al., 2010), which may be due to the polymer purification by dialysis with a higher MWCO membrane $(10,000)$ performed in this work, resulting in a polymer with a different degree of impurities. Anyway, both values are in the range of those presented for typical commercial polysaccharides, such as xanthan and guar gum, 5-50 dL/g (Arvidson, Rinehart, \& Gadala-Maria, 2006). The intrinsic viscosity of fucogel $(16.7 \mathrm{dL} / \mathrm{g})$ was also reported to be within this range (Guetta et al., 2003). The Huggins constant $\left(k_{\mathrm{H}}=0.57\right)$ of the EPS seems to indicate the presence of aggregates, since for flexible macromolecules in a good solvent $k_{\mathrm{H}} \sim 0.3$ (Alves et al., 2010). Fucogel has a similar Huggins constant $\left(k_{\mathrm{H}}=0.55\right)$, confirming some similarity between both fucose-containing polysaccharides in terms of aggregation.

On-going work is focused on the determination of concentration regimes for this particular biopolymer. The results obtained so far show a $C^{*}$ of about $0.09 \mathrm{~g} / \mathrm{dL}$, as such, the intrinsic viscosity was effectively evaluated using polymer concentrations $(0.08-0.01 \mathrm{dL} / \mathrm{g})$ within the diluted regime.

\subsection{Rheological properties in aqueous medium}

The viscosity of the fucose-containing EPS in aqueous medium was compared with several comercial polysaccharides with the same concentration (1.0\%) and solvent $(0.1 \mathrm{M} \mathrm{NaCl})$ (Fig. 3). The flow curve of the fucose-containing EPS solution showed clearly a Newtonian plateau at low shear rates, with a zero shear viscosity approaching 1.0 Pa s, followed by a shear-thining behaviour, with the apparent viscosity decreasing with the increase of shear rate. This behaviour is similar to the one demonstrated by guar gum solution. The solution prepared with fucogel, which is a fucose-containing bacterial exopolysaccharide, also had a similar behaviour under the tested conditions, but the apparent viscosity was somewhat higher. Although xanthan was much more viscous than the EPS for low shear rates, for high shear rates its viscosity was similar to fucogel, guar gum and the EPS solutions. Non-Newtonian 


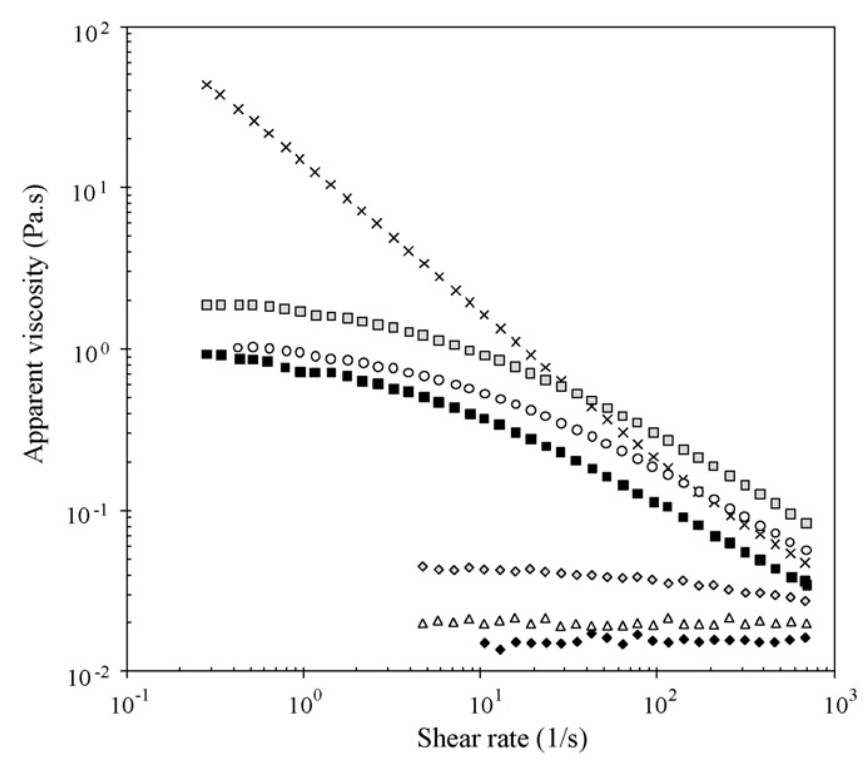

Fig. 3. Comparative flow curves of polysaccharides: fucose-containing $\operatorname{EPS}(\boldsymbol{\square})$, fucogel $(\square)$, xanthan $(\times)$, guar gum $(\bigcirc), \operatorname{CMC}(\diamond)$, pectin $(\triangle)$ and alginate $(\diamond)$.

solutions of polysaccharides are used in several industries, such as food, textile, pharmaceutical and cosmetic, as stabilizing, suspending or thickening agents.

As seen in Fig. 3, there is a clear differentiation between the behaviour of the higher average molecular weights $\left(>10^{6}\right)$ (EPS, xanthan, fucogel and guar gum) and the lower molecular weight polysaccharides $\left(M_{\mathrm{W}} \sim 10^{5}\right)$ (alginate, pectin and $C M C$ ), which were considerably less viscous and had an almost Newtonian behaviour.

The mechanical spectrum of the $1 \%$ EPS aqueous solution is presented in Fig. 4(a). It shows that the loss modulus $\left(G^{\prime \prime}\right)$ is above the storage modulus $\left(G^{\prime}\right)$ in the entire frequency range studied, except at higher frequencies where a cross-over is perceived at a angular frequency of about $17.5 \mathrm{rad} / \mathrm{s}$. Furthermore, the solution obeyed to the Cox-Merz rule (Fig. 4(b)), showing a good superposition of the angular frequency dependence of complex viscosity $\left(\eta^{*}\right)$ with the shear rate dependence of apparent viscosity $(\eta)$. These facts suggest a sample with a viscous behaviour constituted by entangled polymer chains without strong interactions. Fig. 4(a) also shows that the oscillatory data, before and after subjecting the sample to shear rates up to $700 \mathrm{~s}^{-1}$ (corresponding to a shear stress of $20.0 \mathrm{~Pa}$ ), are coincident, revealing a sample with rather stable viscoelastic properties.

\subsection{Emulsion forming and stabilizing capacity}

A preliminary assessment of the fucose-containing EPS potential to be used as an emulsion forming and stabilizer agent was performed with the polymer in aqueous solution ( $0.5 \mathrm{wt}$.\%). Several hydrophobic compounds, namely, food grade oils, mineral oils and hydrocarbons, were assayed (Table 1). For comparison, the same test procedure was performed with Triton X-100, which is a synthetic emulsifier, and other commercial polysaccharides reported to be able to stabilize emulsions, namely, xanthan, pectin and alginate (Leroux, Langendorff, Schick, Vaishnav, \& Mazoyer, 2003; Lim, Kim, Kim, Yoo, \& Kong, 2007). The EPS has proven to possess high emulsion-stabilizing capacity for the food-grade oils tested, namely, sunflower oil, corn oil and rice bran oil, with emulsification indexes of 60,64 and $80 \%$, respectively. The ability of this biopolymer to stabilize emulsions with vegetable oils suggests its potential application as cleaning and emulsifying agent in food industry. Cedar wood oil, though having lower emulsification index (20\%),
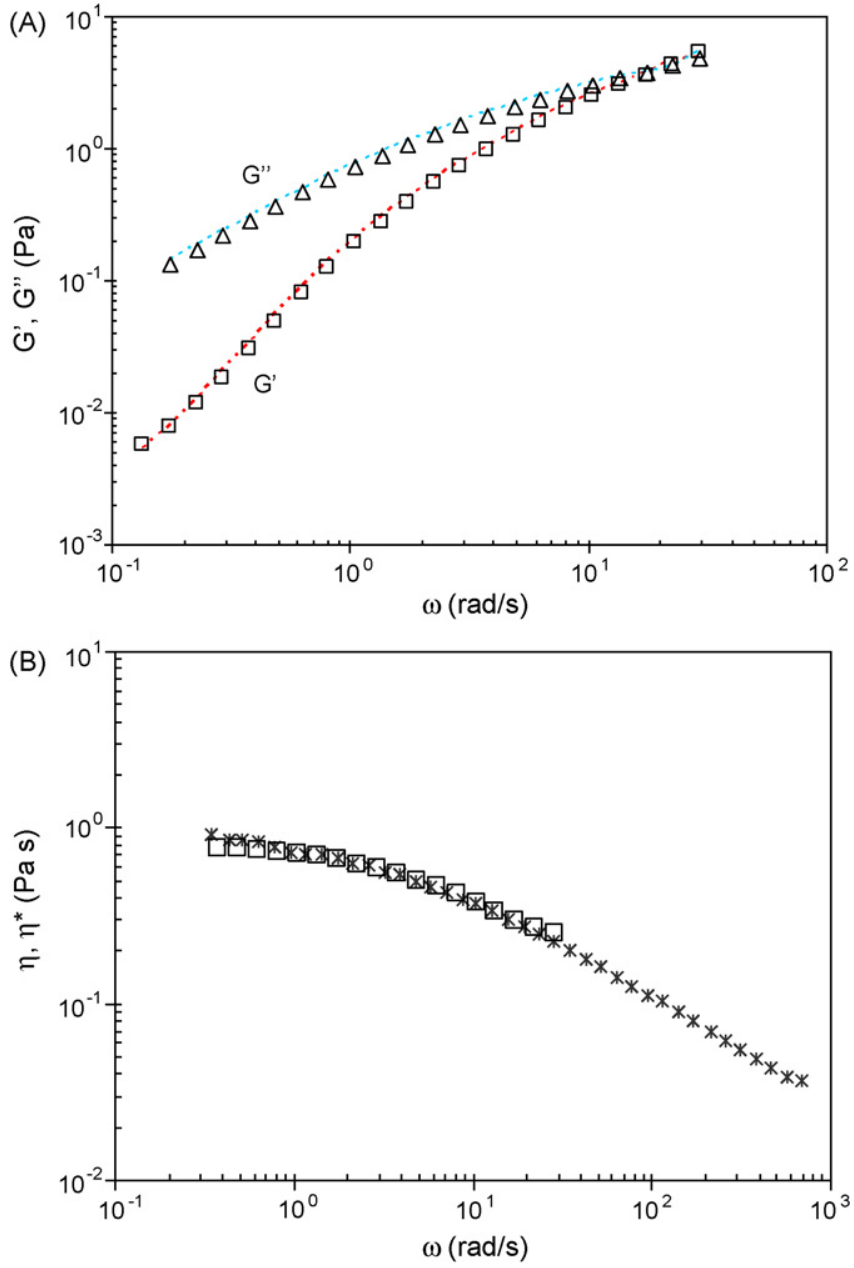

Fig. 4. (a) Mechanical spectrum of a $1 \%(w / w)$ EPS solution. The symbols and the dashed lines correspond to the data obtained before and after subjecting the sample to shear rate values up to $700 \mathrm{~s}^{-1}$, respectively. (b) Apparent viscosity ( $\boldsymbol{X}$ ) and complex viscosity $(\square)$, as a function of the shear rate and angular frequency, respectively.

formed stable emulsions that did not break within the test period. Little emulsion-stabilizing capacity, with emulsions breaking up after only a few minutes, was observed for silicone oil.

The EPS demonstrated to have a lower emulsion forming and stabilizing capacity for hydrocarbons (Table 1 ). In fact, under the conditions tested, no emulsion was formed with xylene, diethylether, benzene or toluene, while low emulsification indexes $(<10 \%)$ were obtained for decane and chloroform. Stable emulsions were formed with hexadecane and hexane, but the emulsification indexes were rather low (22 and 30\%, respectively) (Table 1).

\section{Table 1}

Emulsification index $\left(E_{24}\right)$ for the fucose-containing EPS produced by Enterobacter strain A47 against several hydrophobic compounds in comparison with commercially available emulsion forming and stabilizing agents. All emulsions were prepared by mixing a $0.5 \mathrm{wt}$.\% aqueous solution with each of the hydrophobic compound (3:2, v/v ratio) and left at room temperature for $24 \mathrm{~h}$ to determined $E_{24}$.

\begin{tabular}{llllll}
\hline$E_{24}(\%)$ & EPS & Xanthan & Alginate & Pectin & Triton X-100 \\
\hline Oils & & & & & \\
$\quad$ Cedarwood oil & 20 & 30 & 10 & 40 & 80 \\
$\quad$ Sunflower oil & 60 & 40 & 56 & 20 & 80 \\
$\quad$ Corn oil & 64 & 90 & 40 & 60 & 82 \\
$\quad$ Rice bran oil & 80 & 50 & 50 & 60 & 90 \\
Hydrocarbons & & & & & \\
$\quad$ Hexadecane & 22 & 40 & 10 & 20 & 80 \\
Hexane & 30 & 40 & 10 & 30 & 70 \\
\hline
\end{tabular}


As shown in Table 1, the EPS has demonstrated to have higher emulsification indexes than xanthan, alginate and pectin for most of the compounds tested. Also, the capacity of the fucosecontaining EPS to form and stabilize emulsions is specific for certain hydrophobic compounds, which is also a characteristic of the other commercial polysaccharides tested.

These results show that the fucose-containing EPS has potential to be used as a surface-active compound, which are widely used in the oil and petroleum industries, water and soil bioremediation, metal treatment and processing, detergents and laundry supplies, agriculture, textile manufacturing, pulp and paper processing, paints, cosmetics, pharmaceuticals, personal care products and food processing (Freitas et al., 2009b). Most of the commercially available products, such as Triton X-100, are synthetic, mainly petroleum-derived, generally toxic to the environment and not easily biodegradable. In view of this, natural polymers have emerged as advantageous alternatives because they are biodegradable, less toxic, have improved functionality and have activity under a wider variety of conditions (Banat, Makkar, \& Cameotra, 2000). Moreover, they can be produced from renewable and low cost sources.

\subsection{Flocculating activity}

A preliminary test of the flocculating activity of fucosecontaining EPS was performed in a kaolin suspension and compared with other commercial flocculants, namely, $\mathrm{Al}_{2}\left(\mathrm{SO}_{4}\right)_{3}$, alginate, xanthan, guar gum, chitosan and CMC (Fig. 5). These preliminary tests were performed at room temperature and neutral $\mathrm{pH}$, for flocculants concentration of $0.01 \mathrm{wt} . \%$. As expected, $\mathrm{Al}_{2}\left(\mathrm{SO}_{4}\right)_{3}$ had the highest flocculating activity (30\%), while CMC and xanthan had flocculating activities of 27 and 24\%, respectively. Nevertheless, for the tested conditions, the EPS had similar flocculating activity (28\%), which is a very promising result. Alginate, guar gum and chitosan had much lower flocculating activities ( 11,8 and $6 \%$, respectively) than the EPS (Fig. 5).

These results indicate that the EPS has high flocculating activity, which envisages its potential use for colloid and cell aggregation in several applications, such as water treatment, food and mining industries. Inorganic (e.g. $\left.\mathrm{Al}_{2}\left(\mathrm{SO}_{4}\right)_{3}\right)$ and synthetic organic flocculating agents (e.g. polyacrylamide) are inexpensive products, but have a low biodegradability and are not shear resistant (Singh et al., 2007). On the other hand, some of them are dangerous for human

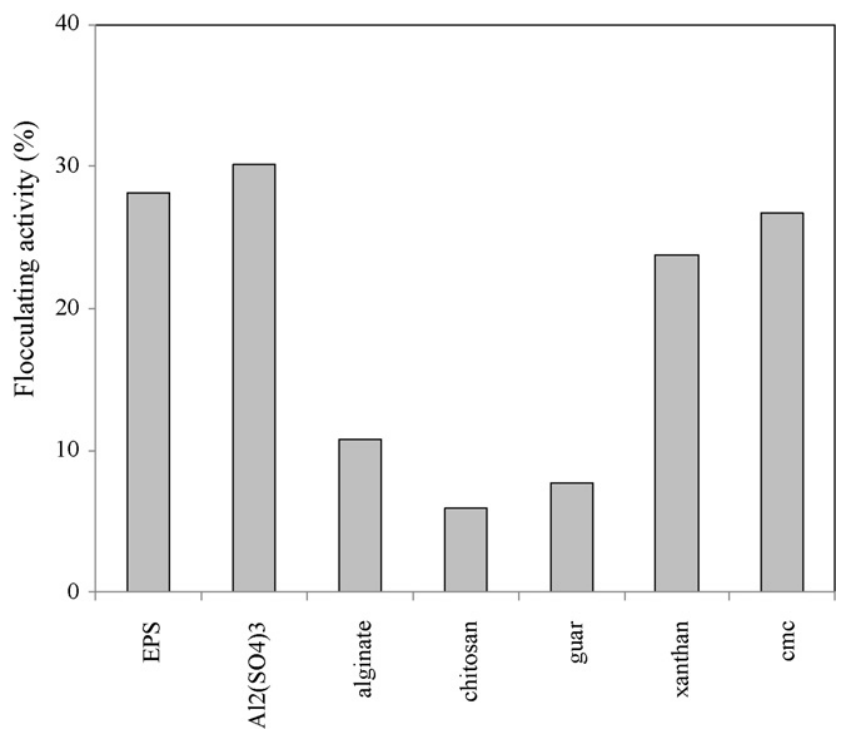

Fig. 5. Flocculating activity of the fucose-containing EPS produced by Enterobacter strain A47 in comparison with commercially available flocculants. health, namely polyacrilamides, whose monomers are neurotoxic, and poly(aluminium chloride) which induces Alzheimer disease. To overcome these environmental and public health problems, naturally occurring flocculants, including several polysaccharides have been suggested as safe alternatives (Freitas et al., 2009b; Lim et al., 2007; Yokoi, Obita, Hirose, Hayashi, \& Takasaki, 2002). The fucose-containing EPS produced by Enterobacter strain A47 may be included amongst these bioflocculants.

\section{Conclusions}

In this work, a fucose-containing biopolymer produced by a newly isolated bacterium, Enterobacter strain A47 (DSM 23139), was described. The biopolymer is a high molecular weight heteropolysaccharide composed of neutral sugars (fucose, galactose and glucose) and acyl groups (pyruvate, succinate and acetate). The FT-IR spectrum of the EPS was in accordance with the composition analysis. The main properties of the EPS, namely, its rheological properties in aqueous medium, emulsion forming and stabilizing capacity, and flocculating capacity, make it a promising alternative to commercial polysaccharides. On-going research includes optimization of the bioreactor production conditions and more detailed studies of the EPS functional properties, envisaging its use in several agro-food, cosmetic, pharmaceutical and industrial applications.

\section{Acknowledgements}

The authors acknowledge SGC Energia, SGPS, SA, Portugal, for supplying the glycerol byproduct from the biodiesel production used in this study. Cristiana A. V. Torres acknowledges Fundação para a Ciência e a Tecnologia, PhD fellowship SFRH/BD/44465/2008.

\section{References}

Alvarez-Mancenido, F., Landin, M., Lacik, I., \& Martínez-Pacheco, R. (2008). Konjac glucomannan and konjac glucomannan/xanthan gum mixtures as excipients for controlled drug delivery systems. Diffusion of small drugs. International Journal of Pharmaceutics, 349, 11-18.

Alves, V. D., Freitas, F., Torres, C. A. V., Cruz, M., Marques, R., \& Grandfils, C. (2010). Rheological and morphological characterization of the culture broth during exopolysaccharide production by Enterobacter sp. Carbohydrate Polymers, 81 758-764.

Arvidson, S. A., Rinehart, B. T., \& Gadala-Maria, F. (2006). Concentration regimes of solutions of levan polysaccharide from Bacillus sp. Carbohydrate Polymers, 65, 144-149.

Banat, I. M., Makkar, R. S., \& Cameotra, S. S. (2000). Potential commercial applications of microbial surfactants. Applied Microbiology Biotechnology, 53, 495-508.

Cescutti, P., Kallioinen, A., Impallomeni, G., Toffanin, R., Pollesello, P., Leisola, M., et al (2005). Structure of the exopolysaccharide produced by Enterobacter amnigenus. Carbohydrate Research, 340, 439-447.

Freitas, F., Alves, V. D., Pais, J., Costa, N., Oliveira, C., Mafra, L., et al. (2009). Characterization of an extracellular polysaccharide produced by a Pseudomonas strain grown on glycerol. Bioresource Technology, 100, 859-865.

Freitas, F., Alves, V. D., Carvalheira, M., Costa, N., Oliveira, R., \& Reis, M. A. M. (2009) Emulsifying behaviour and rheological properties of the extracellular polysaccharide produced by Pseudomonas oleovorans grown on glycerol byproduct. Carbohydrate Polymers, 78, 549-556.

Gong, W.-X., Wang, S.-G., Sun, X.-F., Liu, X.-W., Yue, Q.-Y., \& Gao, B.-Y. (2008). Bioflocculant production by culture of Serratia ficaria and its application in wastewater treatment. Bioresource Technology, 99, 4668-4674.

Grant, W. D., Sutherland, I. W., \& Wilkinson, J. F. (1969). Exopolysaccharide colanic acid and its occurrence in the Enterobacteriaceae. Journal of Bacteriology, 100(3), 1187-1193.

Guetta, O., Mazeau, K., Auzely, R., Milas, M., \& Rinaudo, M. (2003). Structure and properties of a bacterial polysaccharide named Fucogel. Biomacromolecules, 4, 1362-1371.

Harris, L. S., \& Oriel, P. J. (1989). Heteropolysacharide produced by Enterobacter sakazakii. US Patent 4806636.

Hilliou, L., Freitas, F., Oliveira, R., Reis, M. A. M., Lespineux, D., Grandfils, C., et al. (2009). Solution properties of an exopolysaccharide from a Pseudomonas strain obtained using glycerol as single carbon source. Carbohydrate Polymers $78,526-532$.

Krishnan, S., Raj, C. R., Robert, R., Ramanand, A., \& Das, S. J. (2007). Growth and characterization of succinic acid single crystals. Crystal Research and Technology. 42(11), 1087-1090. 
Kurane, R., Takeda, K., \& Suzuki, T. (1986). Screening for and characteristics of microbial flocculants. Agricultural and Biological Chemistry, 50(9), 2301-2307.

Leroux, J., Langendorff, V., Schick, G., Vaishnav, V., \& Mazoyer, J. (2003). Emulsion stabilizing properties of pectin. Food Hydrocolloids, 17(4), 455-462.

Lim, D.-J., Kim, J.-D., Kim, M.-Y., Yoo, S.-H., \& Kong, J.-Y. (2007). Physicochemical properties of the exopolysaccharides produced by marine bacterium Zoogloea sp. KCCM10036. Journal of Microbiology and Biotechnology, 17(6), 979-984.

Moreno, J., Vargas, M. A., Olivares, H., Rivas, J., \& Guerrero, M. G. (1998). Exopolysaccharide production by the cyanobacterium Anabaena sp. ATCC 33047 in batch and continuous culture. Journal of Biotechnology, 60, 175-182.

Paul, F. M. B., Perry, D. F., \& Monsan, P. F. (1996). Strain of Klebsiella pneumoniae, subsp. pneumoniae, and a process for the production of a polysaccharide containing L-fucose. International Patent WO 9623057.

Péterszegi, G., Fodil-Bourahla, I., Robert, A. M., \& Robert, L. (2003). Pharmacological properties of fucose. Applications in age-related modifications of connective tissues. Biomedicine \& Pharmacotherapy, 57, 240-245.

Philbe, J. L. (2002). Nouveau microorganisme de la famille des Enterobacteriaceae. French National Patent FR 2840920.

Rao, M. A. (1999). Rheology of fluids and semisolid foods: Principles and applications. Gaithersburg, MD: Aspen.

Ratto, M., Verhoef, R., Suihko, M.-L., Blanco, A., Schols, H. A., Voragen, A. G. J., et al. (2006). Colanic acid is an exopolysaccharide common to many Enterobacteria isolated from paper-machine slimes. Journal of Industrial Microbiology and Biotechnology, 33, 359-367.
Rinaudo, M. (2004). Role of substituents on the properties of some polysaccharides. Biomacromolecules, 5, 1155-1165.

Singh, R. P., Tripathy, T., Karmakar, G. P., Rath, S. K., Karmakar, N. C., Pandey, S. R., et al. (2007). Novel biodegradable flocculants based on polysaccharides. Current Science, 78(7), 798-803.

Synytsya, A., Copikova, J., Matejka, P., \& Machovic, V. (2003). Fourier transform Raman and infrared spectroscopy of pectins. Carbohydrate Polymers, 54, 97-106.

van den Bulk, R. W., Zevenhuizen, L. P. T. M., Cordewener, J. H. G., \& Dons, J. J. M. (1991). Characterization of the extracellular polysaccharide produced by Clavibacter michiganensis subsp. michiganensis. Phytophatology, 81(6), 619-623.

Vanhooren, P. T., \& Vandamme, E. J. (1999). L-Fucose: Occurrence, physiological role, chemical, enzymatic and microbial synthesis. Journal of Chemical Technology Biotechnology, 74, 479-497.

Vanhooren, P. T., \& Vandamme, E. J. (2000). Microbial production of clavan, an L-fucose rich polysaccharide. In S. Bielecki, J. Tramper, \& J. Polak (Eds.), Food Biotechnology (pp. 109-114). Amsterdam, The Netherlands: Elsevier Science B.V.

Yang, Y. L. (2002). Novel microorganism isolated from Chinese elm (Ulmus sp.) and process for preparing exopolysaccharides by employing the microorganism. US Patent 2002/0115158.

Yokoi, H., Obita, T., Hirose, J., Hayashi, S., \& Takasaki, Y. (2002). Flocculation properties of pectin in various suspensions. Bioresource Technology, 84(3), 287-290. 